\title{
RETOS DE LA ESTILÍSTICA FORENSE EN EL ÁMBITO DEL DISCURSO ELECTRÓNICO DELICTIVO
}

\author{
CHALLENGES IN FORENSIC STYLISTICS IN THE \\ FRAMEWORK OF ONLINE HATE SPEECH OFFENCES
}

\author{
Mario Crespo \\ Universidad de Cádiz, UCA, Cádiz, Espanha
}

\begin{abstract}
Resumen: A pesar de sus beneficios, Internet proporciona una manera accesible, asequible y anónima para la difusión de contenidos ofensivos o discursos de odio. La Lingüística forense cuenta entre su objeto de estudio la atribución de autoría de este tipo de mensajes. Este estudio analiza los factores metodológicos clave que se tienen que considerar en el proceso de identificación de un posible autor. Entre ellos se destacan la selección de los rasgos más apropiados, el tamaño del texto y cómo extraer conclusiones a partir de los datos. Aún queda un largo recorrido en este campo científico para poder solucionar algunos de los problemas relacionados con esta metodología.
\end{abstract}

Palabras clave: Lingüística forense; Reconocimiento de autoría; Discursos de odio electrónicos

Abstract: Despite its benefits, Internet provides an accessible, affordable and anonymous way for the dissemination of offensive contents or hate speeches. Among their object of study Forensic Linguistics includes the authorship attribution of this type of messages. This study looks into the key methodological aspects to be considered in Authorship Attribution. The selection of the more appropriate features, the text size and how to draw conclusions from data are among them. There is still a long way to solve some of the problems related to them in this scientific field.

Keywords. Forensic Linguistics; Authorship Recognition; Online hate speeches

\section{Introducción}

En la era de Internet millones de usuarios disponen de cantidades de información que eran impensables hasta hace varias décadas. Los usuarios no solo acceden a la información sino que interaccionan y promueven libremente nuevos contenidos que están inmediatamente disponibles para el resto de internautas. Sin embargo, a pesar de sus beneficios, Internet provee de un canal accesible, asequible y anónimo para la difusión de contenidos ofensivos o discursos de odio (DJURIC, 2015). Se entiende por discurso de odio cualquier forma de expresión que promueva, incite, extienda o justifique el odio racial, la xenofobia, el antisemitismo o cualquier forma de animadversión o intolerancia (COUNCIL OF EUROPE, 1997). La Lingüística forense, disciplina situada entre Lingüística y el Derecho 
(CICRES BOSCH; GAVALDÀ, 2014) cuenta evidentemente, entre su objeto de estudio, con este tipo de mensajes que pueden llegar a atentar contra los derechos individuales y la convivencia. Será en su vertiente probatoria o evidencial (GIBBONS, 1994), el ámbito que estudie el posible contenido delictivo de este tipo de mensajes o trate de identificar a su autor. Se entiende por atribución de autoría forense la tarea de identificar al autor de un texto cuando se usa el lenguaje como evidencia en un juzgado (OLSSON; LUCHJENBROERS, 2014).

Los discursos de odio electrónicos se caracterizan por el uso medios digitales para la difusión de estos mensajes, incluyendo cualquier dispositivo de acceso a Internet o tecnologías de comunicación como dispositivos móviles (ADL, 2010). Estos discursos están ligados en muchos casos a las redes sociales como Twitter, Facebook o YouTube cuyo crecimiento, amparándose en el anonimato que proveen estos medios, se relaciona también con la actividad organizativa de grupo radicales y extremistas (BERGER; STRATHEARN, 2013; FERRARA, 2016; HANZELKA; SCHMIDT, 2017), con el ciberbulying (BHARGAVA; MEHNDIRATTA; ASAWA, 2013) o más recientemente con campañas de desinformación política promovidas desde grupos interesados en cambiar la intención política de ciudadanos (ROCHA, 2017). Se trata de casos en los que la atribución de autoría puede ser de claro interés tanto para los investigadores policiales como para el proceso judicial tal como indica Grant (2008).

Este estudio analiza las principales variables que se han de tener en cuenta en el proceso de atribución de autoría. En primer lugar, se presenta el concepto de estilo y los fundamentos teóricos que sustentan la identificación del escritor. A continuación se hará un recorrido por la problemática que existe en torno a la selección de rasgos, los requerimientos del corpus para la comparación, y finalmente el procedimiento habitual de expresión de resultados en el proceso judicial.

\section{La atribución de autoría}

El análisis estilístico como enfoque de la atribución de autoría se basa en la premisa de que es posible identificar, describir y medir el estilo individual o idiolecto de un autor a partir de la cuidadosa observación de su conjunto de elecciones lingüísticas (GUILLÉN NIETO, 2008). La escritura es un sistema gráfico para representar y comunicar información. Escribir requiere el desarrollo progresivo de los dos tipos de habilidades: gráficas y lingüísticas. Se trata de dominar una amplia gama de habilidades 
de producción y comprensión (GARTON; PRATT, 1998, apud MCMENAMIN, 2002). Cada vez que un hablante o, en este caso, un escritor emite un mensaje, producirá un texto único e idiosincrático con un determinado número de 'marcadores' y recursos lingüísticos que lo harán irrepetible (TURELL, 2008). Todo esto es posible entendiendo el lenguaje como un sistema de combinación de unidades discretas (MCMENAMIN, 2002) que se combinan unas con otras para crear unidades mayores y con significado.

La tarea de la atribución lingüística no consiste en la identificación de un autor entre millones a partir de la evidencia lingüística, sino más bien en la selección de uno entre un número reducido de candidatos, normalmente inferior a una docena y en muchos casos solo dos (COULTHARD; JOHNSON, 2007). El análisis trata de determinar la consistencia del texto del que se desconoce la autoría o texto 'dubitado', con los rasgos de otros textos de un autor conocido o texto 'indubitado' (OLSSON, 2004). El resultado de la comparación debe ser una opinión fundada que ayudará al proceso legal y, en ningún caso, el resultado final de este.

Existen dos tendencias complementarias en los estudios de atribución de autoría que provienen de ámbitos científicos distintos: ámbito estilísticohumanístico y ámbito computacional-automático. La primera tendencia se ha generado en el ámbito de las humanidades y tiene mayor tradición. Estos estudios de autoría se pueden encontrar ya claramente desde el siglo XVIII (OLSSON, 2004) con la autoría de textos literarios, religiosos o históricos (HOLMES, 1998) (GRANT; BAKER, 2001). Desde esta perspectiva, se describe el proceso de caracterización de estilo como un proceso doble, basado en primer lugar en la identificación de los rasgos que caracterizan al escritor: desviaciones o errores normativos que comete el escritor y variaciones dentro de la norma, o distribuciones preferenciales del emisor en concreto (MCMENAMIN, 2002).

Desde finales de siglo XX y, sobre todo, con el desarrollo de los ordenadores e Internet, aparece un segundo grupo de estudios sobre identificación de emisor basado en técnicas automáticas. Desde este ámbito informático, la atribución de autoría se ha considerado una subclase de los problemas generales asociados a la clasificación automática de textos por el que un sistema trata de determinar la clase más probable a la que pertenece un documento. Esta clase bien puede ser un tema específico, ideología, opinión, grupo social o bien, como en este caso, un posible autor. Estos sistemas aprenden a partir de un conjunto de textos usados de entrenamiento. Para ello primero se computan frecuencias y patrones lingüísticos que se asocian a una determinada clase o autor en particular. 
De esta manera el programa puede aprender cuáles son las características propias de lo que se quiere clasificar y así finalmente tratar de identificar el grupo o clase más probable o cercana de nuevos textos no vistos con anterioridad (AGGARWAL, 2014). Actualmente existe una gran cantidad de estudios sobre identificación de autoría (EL MANAR; KASSOU, 2014; STAMATATOS, 2009; TAMBOLI; PRASAD, 2013; ROCHA, 2017). Desde esta perspectiva computacional, el proceso de atribución de autoría es una tarea en varios pasos: en primer lugar, la limpieza de los elementos innecesarios de los textos de estudio de los autores; un segundo paso de extracción de rasgos de estilo del texto; luego, la construcción de un vector de rasgos que represente a cada escritor estudiado a partir de las características de estilo extraídas; después, el entrenamiento de un algoritmo de clasificación con las características observadas y, finalmente, su puesta en funcionamiento y prueba con textos nuevos de diferentes autores para que intente predecir su autoría (EL MANAR; KASSOU, 2014).

Pareja a la tarea de atribución de autoría, se encuentra la labor de elaboración de perfiles lingüísticos. Un perfil se lleva a cabo cuando ningún sospechoso ha sido identificado y se necesita tener una imagen aproximada de las características sociales del emisor, en este caso del escritor del texto. Tal sería el caso de grabaciones de llamadas telefónicas anónimas de secuestradores o avisos de bombas (WATT, 2010). Su objetivo es reducir la cantidad de posibles sospechosos asociando sus rasgos lingüísticos con ciertos grupos geográficos y sociales. Más allá de los tradicionales estudios lingüísticos de caracterización sociolectal, existe gran atención científica por la elaboración automática de perfiles lingüísticos, destacándose también por el grado de interés el estudio de redes sociales (BAMMAN; EISENSTEIN; SCHNOEBELEN, 2014; DUNN, 2015; NGUYEN, 2013; SCHWARTZ, 2013; STAMATATOS, 2015). Estos trabajos tratan de determinar cualquier tipo de variable social a partir del análisis lingüístico de textos provenientes de medios como Facebook o Twitter.

Dada la naturaleza de este tipo de textos, la tarea de analizar mensajes cortos electrónicos supone varios grandes retos. Bhargava, Mehndiratta y Asawa (2013) destacan cómo los mensajes electrónicos se diferencian de otros tipos de mensajes por ser muy informales, desestructurados y no necesariamente gramaticales, lo que supone un problema a la hora de analizarlos; a esto hay que sumar su tamaño excesivamente cortos (a veces no superior a 140 caracteres).

El siguiente apartado analiza la problemática de la caracterización del estilo de un escritor a partir de los rasgos que éste muestra en sus textos. Como veremos, no existe unanimidad en la ciencia sobre qué rasgos han 
de estudiarse de manera fehaciente para tener una imagen clara del estilo del autor y de si estos se encuentran claramente en todos los textos que este produce. Finalmente veremos las diferencias en los procedimientos empleados por el ámbito humanístico a los usados en los procesos computacionales para la determinación de emisor escrito.

\section{La selección de rasgos relevantes}

En los comienzos de la Lingüística Forense se hablaba del estilo asemejándolo a una "huella dactilar". Si bien es cierto que cada individuo usa el lenguaje de manera diferente, sin embargo esta diferencia interpersonal no se puede observar tan fácilmente como una huella (OLSSON, 2004). Existen más de 1000 potenciales marcadores (PICORNELL GARCÍA, 2012). El lingüista debe considerarlos todos ya que no existe una definición y caracterización consensuada de los marcadores de estilo aceptables.

Todos los estudios sobre autoría trabajan con la misma teoría de idiolecto: recursos lingüísticos y selecciones de rasgos que el autor muestra. El problema radica en distinguir los marcadores idiolectales y cómo evaluarlos (COULTHARD; JOHNSON, 2007). No existe aún una lista clara de características lingüísticas que se puedan analizar para llevar a cabo esta tarea de una manera fidedigna, sin embargo se prefieren aquellas que tengan una gran frecuencia de aparición, que sean inmunes a su supresión voluntaria y que puedan encontrarse en la mayor parte de los individuos de la población de estudio (MCMENAMIN, 2010; PICORNELL GARCÍA, 2012). En la misma línea, Nolan (1983 apud ROSE, 2002) establece que los parámetros de análisis ideales son aquellos que muestren mucha variabilidad entre hablantes y poca variabilidad individual, aquellos que no se puedan modificar voluntariamente y con gran frecuencia de aparición, y finalmente aquellos que sean fácilmente extraíbles y medibles. En este proceso de caracterización de estilo, cobra mucha importancia el concepto de variación inter e intraemisor. Lo que funciona como buen rasgo de estilo para un autor en particular puede no ser satisfactorio para otro (OLSSON, 2004). Además, a veces incluso la cantidad de variación dentro de la obra de un autor, puede ser mayor que la existe entre dos autores concretos. El primer paso hacia el análisis individual de autoría escrita es observar que la variación individual es sistemática, y, finalmente, descubrir su importancia relativa (MCMENAMIN, 2002). Desde esta perspectiva variacionista, la gramática del hablante o escritor demuestra una diferenciación interna ordenada, no aleatoria. 
Desde el ámbito estilístico-humanístico, McMenamin (2002), Olsson (2004) Coulthard y Johnson (2007) destacan como típicos rasgos de análisis el formato del texto, el uso de números y símbolos, las abreviaturas, la puntación, uso de mayúsculas, las faltas de ortografía, el tipo de formación de palabras, las construcciones sintácticas y discursivas, y el uso de frecuencias de palabras y frases. Picornell García (2012) va a hacer distinción entre idiosincrasias de autor y rasgos de estilo. El primero de ellos hace referencia a las marcas o características que solo se encuentran en un autor en particular y que solo lo caracterizan a él. Por el contrario, los rasgos de estilo son elementos de estilo hallados en la mayor parte de escritores, pero el uso que cada uno hace de ellos es diferente, lo que permite contrastarlos. McMenamin (2002) afirma que los marcadores únicos son extremadamente raros, por lo que la atribución de autoría es una suma de rasgos que bien se pueden encontrar en otros autores.

Desde el ámbito computacional, la atribución se autoría se caracteriza por el uso de rasgos cuantitativos. Entre los destacados (GRIEVE, 2007; STAMATATOS, 2009; EL MANAR; KASSOU, 2014; ROCHA, 2017) encontramos, en primer lugar, los basados en grafemas como número de caracteres por palabras, números de elementos en mayúsculas o los gramas de caracteres; entre los rasgos de corte léxico se hallan el número de palabras, el tamaño de palabras, los hapax legomena o los gramas léxicos.

Respecto a los rasgos basados en aspectos sintácticos se observan las clases de palabras, los errores, o el uso de la puntuación o de las palabras de función; en los semánticos, aunque estos son los más escasos en la literatura científica sobre el tema, se encuentran el uso de sinónimos o las dependencias funcionales o semánticas y, finalmente, en los estructurales se analizan, por ejemplo, el número de líneas por párrafo, el número de oraciones, el tamaño léxico de los párrafos o el tamaño léxico de oraciones. Realmente, la extracción automática de rasgos dependerá en gran medida del estado de desarrollo científico de este tipo de analizadores en el campo del Procesamiento del Lenguaje Natural para las lenguas analizadas, lo que explica que aspectos semánticos y pragmáticos sean los menos utilizados en este tipo de trabajos.

Estrechamente ligado con el proceso de selección de los rasgos más adecuados del autor de un texto, se encuentra el tamaño que debe presentar este. En este sentido, hay que preguntarse si un texto determinado es lo suficientemente amplio como para ser capaz de encontrar rasgos en él y extraerlos. Forsyth and Holmes (apud ZHENG, 2006) encuentran muy difícil la tarea de la determinación de escritor por debajo de las 250 palabras 
debido a estos problemas. El siguiente apartado analiza la problemática derivada del tamańo textual y explica brevemente las posturas generales a este respecto.

\section{El tamańo del texto}

Los principales avances actuales en el campo de la atribución de autoría provienen del análisis de textos cortos de emails, redes sociales o mensajería telefónica (JIMÉNEZ, 2012). A este respecto, Coulthard y Johnson (2007) destacan que muchos de estos textos son inferiores a las 200 palabras por lo que la cantidad de rasgos disponibles para analizar va a ser bastante reducida. De igual manera, para Olsson y Luchjenbroers (2014), la identificación del autor se ve obstaculizada por el hecho de que los documentos de trabajo forense (notas de rescate, cartas amenazadoras, etc.) son generalmente demasiado cortas para hacer una identificación fiable. Cuanto más largo es el texto, más fácil es extraer sus rasgos relevantes, pero en el discurso electrónico nos vamos a encontrar con el caso opuesto, los textos son cortos y solo un conjunto reducido de rasgos de estilo va a estar presente (ROCHA, 2017).

Desde el ámbito de las técnicas automáticas se considera que cada texto corto contribuye al proceso de atribución por separado, es decir, es un ejemplo del problema en cuestión. Tales algoritmos de clasificación requieren múltiples instancias de entrenamiento para llegar a un modelo confiable (STAMATATOS, 2009). Desde el ámbito de las humanidades, el uso de estas técnicas automáticas se ve con recelo cuando nos encontramos ante este tamaño tan reducido. Para Olsson (2004), las aproximaciones computacionales tienen graves problemas con textos cortos. En la misma línea, Tiersma (2008) señala que, aunque la identificación del autor es un área muy interesante y potencialmente útil, se ve obstaculizada por el hecho de que los documentos del ámbito forense son generalmente demasiado cortos para hacer una identificación fiable. Tal como indica Queralt (2014), en el proceso de peritaje lingüístico de autoría de textos, una de las primeras tareas que deberá hacer el lingüista forense será el discernir si hay caso lingüístico o no, y esto solo es posible si la muestra es suficientemente larga.

Todavía no se ha establecido claramente un mínimo de palabras adecuado ni cuántos textos son necesarios (STAMATATOS, 2009). A pesar de no existir una longitud mínima probada, resulta evidente que difícilmente se podrán determinar rasgos del autor con un texto anónimo de dos líneas, unas 40 palabras (QUERALT, 2014). Tal como establece Crespo (2017), 
dado que en Lingüística predomina el análisis cualitativo, el enfoque más adecuado debería ser el ajuste muestral basado en proporciones:



$\mathrm{Z} \alpha=$ puntuación crítica $\mathrm{z}$ a un nivel de confianza 1- $\alpha$

$\mathrm{p}=$ probabilidad de éxito, o proporción esperada

$\mathrm{q}=$ probabilidad de fracaso

$\mathrm{d}$ = precisión (error máximo admisible en términos de proporción)

Figura 1: Fórmula estadística para el cálculo del tamaño muestral Fuente: TRIOLA (2013, p. 328)

Asumiendo, por ejemplo, un margen de error al 3\%, un nivel de confianza al 99\% $(\alpha=0,01)$ de acuerdo con estándares estadísticos normales y un $\mathrm{p}=0.5$ que maximiza el tamaño muestral, se obtendría un tamaño textual aproximado de 1843. Sin embargo, esta formulación sería insuficiente para fenómenos lingüísticos muy infrecuentes, lo cual es bastante habitual al estudiar el léxico y determinados elementos sintácticos y textuales. Eder (2015), tras un proceso de experimentación en atribución con diferentes lenguas, sitúa independientemente de la lengua, el tamaño adecuado en torno a las 5000 palabras con el uso principalmente de gramas de caracteres y palabras.

A pesar de los problemas, el estudio de atribución con textos cortos es bastante recurrente en la literatura científica (ROCHA, 2017) y se está probando con diferentes lenguas: Abbasi y Chen (2005) para el árabe y el inglés, Bhargava, Mehndiratta y Asawa (2014) y Bobicev (2013) para el inglés, Cristani (2012) para el italiano, Zheng (2006) para el inglés y el chino, Rico Sulayes (2012) para el español o Silva (2012) para el portugués. Los resultados difieren en sus grados de precisión dependiendo del algoritmo de clasificación, del rasgo usado o del tamaño del corpus disponible, superando en pocos casos el $85 \%$ de precisión.

Finalmente, hay que destacar que en la mayor parte de los estudios de atribución de autoría nos encontramos con corpus creados ad hoc en 
los que se experimenta, como hemos visto, usando cierto tipo de variables y observando la tasa de éxito al clasificar textos. Estos corpus pueden ayudarnos a evaluar las técnicas, pero en muchos casos distan de la situación normal que uno se podría encontrar judicialmente. Olsson (2004) señala que es baste común que los lingüistas forenses apenas trabajen con solo dos o tres textos de no más de cien palabras de extensión y que sea imposible contar con más textos de referencia para poder hacer comparaciones. Además, tal como señala Rocha (2017), en atribución de autoría no siempre es posible suponer que los textos usados de entrenamiento y los usados de evaluación compartan las mismas propiedades. Esto ocurre porque, a veces, para poder hacer comparaciones usamos como referencia textos procedentes de géneros discursivos diferentes a los dubitados y que, por lo tanto, no están reflejando adecuadamente las características del documento sobre el que se realiza la pericial lingüística. Se necesitan modelos de atribución de autoría que tengan buen rendimiento aunque nos encontremos con géneros y temas diferentes. Sin duda, este es un aspecto que deberá ser estudiado a fondo en el futuro.

\section{La expresión de resultados en atribución de autoría}

El análisis forense trata de determinar, más allá de toda duda, la consistencia de los rasgos respecto a un determinado autor. En estos casos, los lingüistas forenses acuden ante el tribunal para exponer y defender las conclusiones de sus análisis y contribuir a la obtención de una resolución inculpatoria o exculpatoria según el caso. Existe una serie de requerimientos legales que varían en función del sistema judicial (OLSSON, 2004). Aunque el sistema legal español permite que la interpretación de la evidencia científica se evalúe libremente, la tendencia general pericial es adaptarse a lo que se conoce como sistema "Daubert" (ECKERT; WRIGHT, 1997) que establece una serie de criterios para valorar la admisibilidad de las pruebas científicas en el proceso penal (DE LUCA; NAVARRO; CAMERIERE, 2013, s.p.):

a) La controlabilidad y falseabilidad de la teoría científica sobre la que se basa la prueba;

b) La determinación del porcentaje de error, así como el cumplimiento de los estándares correspondientes a la técnica empleada;

c) La existencia de un control ejercido por expertos a través de la peer review o revisión por pares en las revistas científicas con impacto;

d) La existencia de un consenso general en la comunidad científica de referencia.

fragmentum, n. 50, Jul./Dez. 2017. 
Sin embargo, la mayoría de los lingüistas forenses y especialistas del campo se han sentido tradicionalmente incapaces de expresar sus hallazgos en términos de probabilidades e incluso no dan ninguna indicación sobre su validez más allá de toda duda razonable (COULTHART; JOHNSON, 2007). Esto ha provocado que la Lingüística esté fuertemente estigmatizada y se suelen cuestionar sus resultados (PÉREZ GONZÁLEZ, 2005).

La forma más habitual de expresión de resultados en Lingüística forense son las escalas de certeza que se basan tanto en el análisis como en la pericia y en el saber del perito. Una vez analizada la evidencia y comparados los textos, el experto escogerá el valor que más se aproxime a su juicio personal. Existen diferentes tipos de escalas de certeza, la figura 2 muestra la propuesta de Carlos Delgado (2001):

NIVEL DE IDENTIFICACIÓN
NIVEL DE ALTA PROBABILIDAD
NIVEL MEDIO-ALTO DE PROBABILIDAD
NIVEL INCONCLUSIVO
NIVEL MEDIO-BAJO DE PROBABILIDAD
NIVEL DE BAJA PROBABILIDAD
NIVEL DE ELIMINACIÓN

Figura 2: Escala de expresión de resultados Fuente: DELGADO (2001, p. 244).

Entre los problemas del uso de este tipo de escalas 'semánticas' se encuentra, en primer lugar, la posible subjetividad derivada de su aplicación ya que no existen criterios totalmente objetivos para seleccionar un determinado nivel o etiqueta, y, en segundo lugar, el hecho de que no se puede estar totalmente seguro de que jueces y jurados le atribuyan el mismo significado que los expertos le dieron inicialmente (COULTHART; JOHNSON, 2007).

Desde el ámbito computacional de autoría, el resultado del sistema se expresa bien en forma de valores booleanos (clasificador binario), bien mediante la probabilidad de pertenecer a una determinada categoría (en este 
caso un 'autor') o bien a través de una lista jerarquizada de clases ordenadas en función de cuál de una ellas es la que más se ajusta a lo observado (SAVOY, 2016). Estos resultados, aunque muy útiles, no permiten cuantificar tampoco el grado de certeza que se tiene de que el texto en cuestión satisfaga las características estilísticas propias de un autor más allá de toda duda. Además, en los sistemas computacionales no se contempla la determinación de los mínimos de calidad y extensión de las evidencias para permitir un mínimo de fiabilidad a la hora de expresar la opinión sobre ellas.

Rose (2002) establece que el lingüista forense debe indicar tanto la probabilidad de la hipótesis o probabilidad de que la evidencia analizada pueda ocurrir tanto para el caso de que el acusado sea el emisor o el escritor como que no lo sea, así como la fuerza de esta evidencia. Recordemos que la probabilidad de declarar culpable al acusado es competencia exclusiva de los jueces. No llegar a ninguna atribución es mejor que una atribución falsa (EDER, 2015), aspecto que no suele estar considerado en este tipo de sistemas automáticos. Un procedimiento de expresión de resultados que trata de satisfacer los requerimientos anteriores es la razón de verosimilitud. A grandes rasgos se trata del cociente entre la probabilidad de la acusación y la probabilidad de la defensa (H0, la hipótesis nula) y expresa el grado en el que la evidencia apoya la hipótesis de la acusación (ROSE, 2002). Si bien es cierto, que aún queda un largo camino en su uso debido a que los lingüistas no saben cuantificar adecuadamente sus hallazgos, lo que hace que suela reportar más confusión que claridad (COULTHARD; JOHNSON, 2007).

\section{Conclusiones}

En este artículo, hemos podido ver cuáles son los principales problemas a los que se enfrenta la Lingüística forense al trabajar con textos electrónicos. Existe una preocupación social ante el aumento de mensajes anónimos en redes sociales o foros de Internet que reflejan discursos de odio, lo que en algunos casos pueden llegar atentar contra los derechos individuales, el ciberbullying, o la organización y difusión de contenidos procedentes de grupos delictivos a través de estos medios. En estos casos, se hace necesario contar con herramientas capaces de determinar la autoría o establecer las características sociales de los usuarios que los publican. Tal vez porque es mucho más fácil eliminar a alguien como sospechoso con un grado de confianza razonable que demostrar su culpabilidad fuera de toda duda razonable, no es de extrañar que la mayor parte de la experiencia lingüística en casos penales haya sido para la defensa (TIERSMA, 2008). 
Como hemos visto, existen dos tendencias generales en reconocimiento de autoría: humanística y computacional. Ambas se necesitan mutuamente, ya que aportan perspectivas y conocimientos complementarios que pueden ayudar a un mejor esclarecimiento de la posible autoría de un determinado texto. Todavía queda por descubrir qué características lingüísticas son indicadores confiables de la autoría, y cuán fiables son esas características. Rocha (2017) señala algunas consideraciones claves para la atribución forense de la autoría en el futuro:

- Mejor control del conjunto de pruebas sobre el que se harán las predicciones, que podría limitarse a una sola muestra.

- Mejor control sobre la calidad de los datos de análisis utilizados como corpus de entrenamiento o corpus indubitado.

- La necesidad de un proceso bien definido. Esto es necesario para algoritmos precisos y eficientes, así como consideración legal.

- La determinación de una tasa de error bien definida para un algoritmo, antes de que se aplique a un problema del mundo real.

- Posible potencial malicioso o posibilidad de que un autor bajo investigación evite deliberadamente los métodos de atribución.

La investigación continúa, y la progresiva disponibilidad de corpus grandes con diferentes muestras de escritura, hará que se pueda avanzar en el futuro (aunque el tamaño típicamente pequeño de los documentos en la mayoría de los casos criminales siempre será un problema). La investigación en curso hará que los enfoques lingüísticos forenses sean más confiables y que este campo puede ser cada vez más útil para los investigadores y fiscales.

\section{Referencias}

ABBASI, Ahmend; CHEN, Hsinchun. Applying authorship analysis to extremist-group web forum messages. Intelligent Systems, IEEE, n. 20, v. 5, p. 67-75. 2005 .

ADL. Responding to Cyber hate. Toolkit for Action. Anti Defamation League, 2010. Disponible en: <http://www.adl.org/assets/pdf/combatinghate/ADL-Responding-to-CyberhateToolkit.pdf>. Acceso en: 15 de julio de 2017. 
AGGARWAL, Charu C. Data classification: algorithms and applications. New York: CRC Press, 2014.

BAMMAN, David; EISENSTEIN, Jacob; SCHNOEBELEN, Tyler. Gender identity and lexical variation in social media. Journal of Sociolinguistics, n. 18, v. 2, p. 135-160, 2014.

BHARGAVA, Mudit; MEHNDIRATTA, Pulkit; ASAWA, Krishna. Stylometric analysis for authorship attribution on twitter. In: International Conference on Big Data Analytics. Springer, Cham, 2013, p. 37-47.

BERGER, John. M; STRATHEARN, Bill. Who Matters Online: Measuring influence, evaluating content and countering violent extremism in online social networks. Int. Centre for the Study of Radicalisation. King's College London, 2013.

CICRES BOSCH, Jordi; GAVALDÀ, Nuria. La lingüística forense: la llengua com a evidència. Revista de Llengua i Dret, n. 61, p. 60-71, 2014.

COULTHARD, M Malcolm; JOHNSON Alison. An Introduction to Forensic Linguistics: Language in Evidence. London and New York: Routledge, 2007.

COUNCIL OF EUROPE. Committee of Ministers. Recommendation 97 (20) on "hate speech", 1997.

CRESPO, Mario. Corpus ForenUCA: diseńo, objetivos y estado actual en el marco del Instituto de Investigación en Lingüística Aplicada. Actas... XXXV Congreso Internacional de AESLA. Asociación Española de Lingüística Aplicada, 2017.

CRISTANI, Marco et al. Conversationally-inspired stylometric features for authorship attribution in instant messaging. Proceedings of the 20th ACM international conference on Multimedia, p. 1121-1124, 2012.

DE LUCA, Stefano; NAVARRO, Fernando; CAMERIERE, Roberto. La prueba pericial y su valoración en el ámbito judicial español. Revista Electrónica de Ciencia Penal y Criminología, n. 15, p. 1-19, 2013.

DELGADO ROMERO, Carlos. La identificación de locutores en el ámbito forense. Tesis doctoral. Universidad Complutense de Madrid, 2001.

DJURIC, N. et al. Hate speech detection with comment embeddings. In: Proceedings of the 24th International Conference on World Wide Web, p. 29-30. ACM. 2015. 
DUNN, Jonathan et al. Digital Scholarship in the Humanities, n. 31, v. 4, p. 689-710, 2016.

ECKERT, William G.; WRIGHT, Ronald K. Scientific evidence in court. In: William G. Eckert (Ed.), Introduction to forensic sciences. New York: CRC Press, 1997.

EDER, Maciej. Does size matter? Authorship attribution, small samples, big problem. Digital Scholarship in the Humanities, n. 30, v. 2, p. $167-$ $182,2015$.

EL MANAR, El Bouanani; KASSOU, Sara; KASSOU, Ismail. Authorship analysis studies: A Survey. International Journal of Computer Applications, n. 86, v. 12, p. 22-29, 2014.

FERRARA, Emilio et al. Predicting online extremism,content adopters, and interaction reciprocity. SocInfo 2016: 8th International Conference on Social Informatics, 2016.

GIBBONS, John. (Org.). Language and the Law. Londres y Nueva York: Lognman. 1994.

GRANT, Tim D.; BAKER, Kevin L. Identifying reliable, valid markers of authorship: a response to Chaski. Forensic Linguistics, 8, p. 66-79, 2001.

GRANT, Tim. Approaching questions in forensic authorship analysis. In GIBBONS, John; TURELL, María Teresa. Dimensions of forensic linguistics. Vol. 5. John Benjamin’s Publishing. 2008.

GRIEVE, Jack. Quantitative authorship attribution: An evaluation of techniques. Literary and linguistic computing, n. 22, v. 3, p. 251-270, 2007.

GUILLÉN NIETO, Victoria et al. Exploring State-Of-Art Software for Forensic Authorship Identification. International Journal of English Studies, North America, 8, 2008.

HANZELKA, Jan; SCHMIDT, Ina. Dynamics of Cyber Hate in Social Media: A Comparative Analysis of Anti-Muslim Movements in the Czech Republic and Germany. International Journal of Cyber Criminology, n. 11, v. 1, 2017.

HOLMES, David I. The evolution of stylometry in humanities scholarship. Literary and linguistic computing, n. 13, v. 3, p. 111-117, 1998.

JIMÉNEZ, Miriam. La Lingüística forense: licencia para investigar la lengua. In: GARAYZÁBAL, Elena; JIMÉNEZ, Miriam; REIGOSA, 
Mercedes. Lingüística forense: la lingüística en el ámbito legal y policial. Madrid: Euphonia Ediciones, 2012.

MCMENAMIN, Gerald. R. Forensic linguistics: Advances in forensic stylistics. CRC press, 2002.

NGUYEN, Dong et al. 'How old do you think I am?' A study of language and age in Twitter. Proceedings of the Seventh International AAAI Conference on Weblogs and Social Media, ICWSM 2013, Palo Alto, CA, AAAI Press, p. 439-448, 2013.

OLSSON, John. Forensic Linguistics. An Introduction to Language, crime and the law. Continuum, 2004.

OLSSON, John; LUCHJENBROERS, June. Forensic Linguistics. Bloomsbury. 2014.

PÉREZ GONZÁLEZ, Luis. Perspectivas de desarrollo de peritaje lingüístico en España. In: TURELL, María Teresa (Org.). Lingüística forense, lengua y derecho, 2005.

PICORNELL GARCÍA, Isabel. La aplicación de atribución de autoría en la investigación e inteligencia La aplicación práctica. In: GARAYZÁBAL, Elena; JIMÉNEZ, Miriam; REIGOSA, Mercedes. Lingüística forense: la lingüística en el ámbito legal y policial. Madrid: Euphonia Ediciones, 2012.

QUERALT, Sheila. Acerca de la prueba lingüística en atribución de autoría hoy. Revista de Llengua i Dret, n. 62, 2014.

RICO SULAYES, Antonio. Quantitative Authorship Attribution of Users of Mexican Drug Dealing Related Online Forums. Dissertations/ Theses - Doctoral Dissertations. Georgetown University, 2012.

ROCHA, Anderson et al. Authorship attribution for social media forensics. IEEE Transactions on Information Forensics and Security, n. 12, v. 1, p. 5-33, 2017.

ROSE, P. Forensic speaker identification. CRC Press, 2002.

SAVOY, Jacques. Estimating the probability of an authorship attribution. Journal of the Association for Information Science and Technology, n. 67, v. 6, p. 1462-1472, 2016.

SCHWARTZ, Andrew H. et al. Personality, gender, and age in the language of social media: The open-vocabulary approach. PloS one, n. 8, v. 9, p. 7391, 2013. 
SILVA, Rui. Sousa et al. Twazn me!!! (authorship analysis of micro-blogging messages). In: R. MUÑOZ, Andres; MONTOYO, Andrés; MÉTAIS, Elisabeth; Natural Language Processing and Information Systems. Springer Berlin Heidelberg, p. 161-168, 2012.

STAMATATOS, Efstathios. A survey of modern authorship attribution methods. Journal of the American Society for information Science and Technology, n. 60, v. 3, p. 538-556, 2009.

STAMATATOS, Efstathios et al. Overview of the PAN/CLEF 2015 Evaluation Lab, Experimental IR Meets Multilinguality, Multimodality, and Interaction. Lecture Notes in Computer Science, 9283, NY: Springer International Publishing, p. 518-538, 2015.

TAMBOLI, Mubin Shaukat; PRASAD, Rajesh S. Authorship Analysis and Identification Techniques: A Review. International Journal of Computer Applications, n. 77, v. 16, 2013.

TIERSMA, Peter. What is forensic linguistics? Aston University Centre for Forensic Linguistics. 2008. Disponible en: <http://www.languageandlaw. org/FORENSIC.HTM>. Acceso en: 13 jul. 2017.

TRIOLA, MARIO F. Estadística. Addison-Wesley, 2009.

TURELL, María; PLAGIARISM, Teresa. In: J. Gibbons, M. T. Turell, Dimensions of forensic linguistics. Vol. 5. John Benjamins Publishing, 2008.

WATT, Dominic. The Identification of the Individual through Speech. Language and Identities, Edinburgh: Edinburgh University, p. 76-85. 2010.

ZHENG, Rong et al. A framework for authorship identification of online messages: Writing-style features and classification. Journal of the American Society for Information Science and Technology, n. 57, v. 3, p. 378-393, 2006. 